TRANSACTIONS OF THE

AMERICAN MATHEMATICAL SOCIETY

Volume 357, Number 10, Pages 3933-3951

S 0002-9947(05)03981-4

Article electronically published on May 10, 2005

\title{
THE UNIFORM COMPANION FOR LARGE DIFFERENTIAL FIELDS OF CHARACTERISTIC 0
}

\author{
MARCUS TRESSL
}

\begin{abstract}
We show that there is a theory UC of differential fields (in several commuting derivatives) of characteristic 0 , which serves as a model companion for every theory of large and differential fields extending a model complete theory of pure fields. As an application, we introduce differentially closed ordered fields, differentially closed p-adic fields and differentially closed pseudofinite fields.
\end{abstract}

\section{INTRODUCTION}

We introduce a first order theory of differential fields of characteristic 0 , in $K$ commuting derivatives, called UC (for Uniform Companion), with the following properties:

(I) Whenever $L$ and $M$ are models of UC and $A$ is a common differential subring of $L$ and $M$ such that $L$ and $M$ have the same universal theory over $A$ as pure fields, then they have the same universal theory over $A$ as differential fields. Here, two structures $M$ and $L$ of the same signature with a common substructure $A$ have the same universal theory over $A$ if every universal sentence with parameters from $A$ holds in $M$ if and only if it holds in $L$.

(II) Every differential field $F$ which is 'large' can be extended to a model of UC, and this extension is elementary in the language of rings. Here, a (pure) field $F$ is called large if every smooth curve defined over $F$ that has an $F$-rational point also has infinitely many $F$-rational points (for example, every PAC-field, every PRC-field, every PpC-field and every field admitting a nontrivial henselian valuation is large).

This is our Main Theorem 6.2 below. The theory UC is a simultaneous axiomatization of differentially closed fields (introduced in [Bö], [McG]) and of differentially closed ordered fields (introduced by M. Singer (cf. [Si]) in the case of one derivative). If we add $\mathrm{UC}$ to the theory of p-adically closed fields in the language of p-valued fields, we get the model completion of p-adically closed, differential fields; this theory also has quantifier elimination. If we add UC to the theory of pseudo finite fields in the language of rings enlarged by some constants, we get the model completion of pseudo finite, differential fields (of characteristic 0 ). This is explained in section 8

Received by the editors April 22, 2003.

2000 Mathematics Subject Classification. Primary 03C65, 12H05; Secondary 03C10, 13N99.

Key words and phrases. Differential algebra, differentially closed, large field, model theory, model complete.

(c)2005 American Mathematical Society Reverts to public domain 28 years from publication 3933 
More generally, properties (I) and (II) of UC above, imply that for every model complete theory $T$ of large fields in the language of rings, the theory $T \cup \mathrm{UC}$ of differential fields is model complete. Moreover, if this is the case, $T \cup U \mathrm{UC}$ is complete if $T$ is complete and $T^{*} \cup \mathrm{UC}$ has quantifier elimination if a definable extension $T^{*}$ of $T$ has quantifier elimination (cf. Theorem 7.2, Theorem 7.1 contains the full model theoretic consequence of properties (I) and (II)).

The theory of UC is inductive (i.e. axiomatized by $\forall \exists$-sentences), and it is called "the" uniform companion for large differential fields, since UCU "large fields" is the unique, inductive theory of large differential fields satisfying properties (I) and (II) above (cf. Proposition 6.3).

The axioms of UC say that certain systems of differential equations in $K$ derivatives and $N$ differential indeterminates $(K, N \in \mathbb{N})$ are solvable. These systems are called algebraically prepared systems (cf. 3.1] and the end of section 3).

Briefly, an algebraically prepared system is characterized by the following two properties:

a. The solvability of the system in a differential field extension of the given field can be reduced to an algebraic geometric problem when we view the system as a system of ordinary polynomials.

b. The system has a regular solution in the given field when we view the system as a system of ordinary polynomials.

Then, if the differential field is large (i.e. it is large as a pure field), condition $\mathrm{b}$ implies that the solutions of the given systems (viewed as a system of ordinary polynomials) in $F$ are Zariski dense in the variety defined by the system (cf. [5.3). With this information, condition a guarantees a differential solution of the system in a differential field extension of $F$ which is an elementary extension of the pure field $F$.

\section{Differential algebraic PReliminaries}

In this section we recall notions from basic differential algebra; mainly we explain what a characteristic set is in the differential setup. Our main source here is Kolchin's book [Ko] on differential algebra and algebraic groups.

Let $R$ be a differential ring in $K$ pairwise commuting derivatives $\partial_{1}, \ldots, \partial_{K}$. Let $Y:=\left(Y_{1}, \ldots, Y_{N}\right)$ be a tuple of $N$ indeterminates over $R$ and let

$$
\mathcal{D}:=\left\{\partial_{1}^{i_{1}} \ldots \partial_{K}^{i_{K}} \mid i_{1}, \ldots, i_{K} \in \mathbb{N}_{0}\right\}
$$

be the free abelian monoid generated by $\left\{\partial_{1}, \ldots, \partial_{K}\right\}$, which we denote multiplicatively. For each $\Theta \in \mathcal{D}$ and $n \in\{1, \ldots, N\}$ let $\Theta Y_{n}$ be an indeterminate, where $\Theta Y_{n}=Y_{n}$ if $\Theta=\partial_{1}^{0} \ldots \partial_{K}^{0}$ by definition. Moreover let

$$
\mathcal{D} Y:=\left\{\Theta Y_{n} \mid \Theta \in \mathcal{D}, 1 \leq n \leq N\right\} .
$$

The differential polynomial ring over $R$ in $K$ derivatives and $N$ indeterminates is the polynomial ring $R\{Y\}:=R[y \mid y \in \mathcal{D} Y]$ together with the uniquely determined derivations $\partial_{i}$ such that $\partial_{i}\left(r \cdot \Theta Y_{n}\right)=\left(\partial_{i} r\right) \cdot \Theta Y_{n}+r \cdot\left(\partial_{i} \Theta\right) Y_{n}(1 \leq i \leq K 1 \leq n \leq$ $N, r \in R)$. So $R\{Y\}$ is a differential ring extension of $R$ and $R\{Y\}$ is the free object generated by $N$ elements over $R$ in the category of differential rings with $K$ commuting derivatives. The set of all powers of variables from $\mathcal{D} Y$ is denoted by

$$
\mathcal{D} Y^{*}:=\left\{y^{p} \mid y \in \mathcal{D} Y, p \in \mathbb{N}\right\} .
$$


(2.1) Definition. The rank on $\mathcal{D} Y^{*}$ is the map rk: $\mathcal{D} Y^{*} \longrightarrow \mathbb{N}_{0} \times\{1, \ldots, N\} \times$ $\mathbb{N}_{0}^{K} \times \mathbb{N}$ defined by

$$
\operatorname{rk}\left(\partial_{1}^{i_{1}} \ldots \partial_{K}^{i_{K}} Y_{n}\right)^{p}:=\left(i_{1}+\ldots+i_{K}, n, i_{K}, \ldots, i_{1}, p\right) .
$$

The set $\mathcal{O}:=\mathbb{N}_{0} \times\{1, \ldots, N\} \times \mathbb{N}_{0}^{K} \times \mathbb{N}$ equipped with the lexicographic order (hence the first component is the dominating one) is well ordered. Note that the order type of the image of rk in $\mathcal{O}$ is the order type of $\mathbb{N}$.

(2.2) Definition. We say a variable $y \in \mathcal{D} Y$ appears in $f \in R\{Y\}$ if $y$ appears in $f$ considered as an ordinary polynomial (hence $Y_{1}$ does not appear in $\partial_{1} Y_{1}$ ). The leader $u_{f}$ of $f \in R\{Y\} \backslash R$ is the variable $y \in \mathcal{D} Y$ of highest rank which appears in $f$. Moreover $u_{f}^{*}:=u_{f}^{\operatorname{deg}_{u_{f}} f} \in \mathcal{D} Y^{*}$ denotes the highest power of $u_{f}$ in $f$. We extend the rank to polynomials $f \in R\{Y\}$ by

$$
\operatorname{rk}(f):=\operatorname{rk}\left(u_{f}^{*}\right) \in \mathcal{O} .
$$

(2.3) Definition. If $g, f \in R\{Y\}, g \notin R$, are polynomials, then $f$ is called weakly reduced with respect to $g$ if no proper derivative of $u_{g}$ appears in $f$. $f$ is called reduced with respect to $g$ if $f$ is weakly reduced with respect to $g$ and if $\operatorname{deg}_{u_{g}} f<$ $\operatorname{deg}_{u_{g}} g$.

The polynomial $f$ is called (weakly) reduced with respect to a nonempty set $G \subseteq R\{Y\} \backslash R$ if $f$ is (weakly) reduced with respect to every $g \in G$.

A nonempty subset $G \subseteq R\{Y\} \backslash R$ is called autoreduced if every $f \in G$ is reduced with respect to all $g \in G, g \neq f$. If $G$ consists of a single element, then $G$ is called autoreduced as well.

It easy to see that $u_{f} \neq u_{g}$ - hence $\operatorname{rk} f \neq \operatorname{rk} g$ - if $f, g$ are different polynomials from an autoreduced set. Moreover, by [Ko], Chap. O, Section 17, Lemma 15(a), we have

(2.4) Proposition. Every autoreduced set is finite.

Let $\infty$ be an element bigger than every element in $\mathcal{O}$ and let $(\mathcal{O} \cup\{\infty\})^{\mathbb{N}}$ be equipped with the lexicographic order. We define the rank of an autoreduced set $G$ to be an element of $(\mathcal{O} \cup\{\infty\})^{\mathbb{N}}$ as follows. Let $G=\left\{g_{1}, \ldots, g_{l}\right\}$ with rk $g_{1}<\ldots<$ $\operatorname{rk} g_{l}$. Then

$$
\operatorname{rk} G:=\left(\operatorname{rk} g_{1}, \ldots, \operatorname{rk} g_{l}, \infty, \infty, \ldots\right) .
$$

(2.5) Proposition. There is no infinite sequence $G_{1}, G_{2}, \ldots$ of autoreduced sets with the property $\mathrm{rk} G_{1}>\operatorname{rk} G_{2}>\ldots$

Proof. [Ko], Chap. I, Section 10, Proposition 3.

(2.6) Definition. If $M \subseteq R\{Y\}$ is a set not contained in $R$, then by Proposition 2.5 the set $\{\operatorname{rk} G \mid G \subseteq M$ is autoreduced $\}$ has a minimum. Every autoreduced subset $G$ of $M$ with this rank is called a characteristic set of $M$.

(2.7) Proposition. If $G$ is a characteristic set of $M \subseteq R\{Y\}$ and $f \in M \backslash R$, then $f$ is not reduced with respect to $G$.

Proof. If $f \in M \backslash R$ is reduced with respect to $G$, then the set $\{g \in G \mid \operatorname{rk} g<$ $\operatorname{rk} f\} \cup\{f\}$ is an autoreduced subset of $M$ of rank strictly lower than the rank of $G$, which is impossible. 
From now on we assume that $R$ is a differential domain in $K$ derivatives containing $\mathbb{Z}$.

(2.8) Definition. Let $f \in R\{Y\} \backslash R, f=f_{d} u_{f}^{d}+\ldots+f_{1} u_{f}+f_{0}$ with polynomials $f_{d}, \ldots, f_{0} \in R\left[y \in \mathcal{D} \mid y \neq u_{f}\right]$ and $f_{d} \neq 0$. The initial $I(f)$ of $f$ is defined as

$$
I(f):=f_{d} .
$$

The separant $S(f)$ of $f$ is defined as

$$
S(f):=\frac{\partial}{\partial u_{f}} f=d \cdot f_{d} u_{f}^{d-1}+\ldots+f_{1} .
$$

Moreover, for every subset $G=\left\{g_{1}, \ldots, g_{l}\right\}$ of $R\{Y\} \backslash R$ we define

$$
H(G):=\prod_{i=1}^{l} I\left(g_{i}\right) \cdot S\left(g_{i}\right) \text { and } H_{G}:=\left\{\prod_{i=1}^{l} I\left(g_{i}\right)^{n_{i}} S\left(g_{i}\right)^{m_{i}} \mid n_{i}, m_{i} \in \mathbb{N}_{0}\right\} .
$$

Since $R$ is a domain and $\mathbb{Z} \subseteq R$, the set $H_{G}$ does not contain 0. Moreover, $S(g)$ and $I(g)$ are reduced with respect to $G(g \in G)$, if $G$ is an autoreduced set.

(2.9) Theorem. Let $G \subseteq R\{Y\}$ be an autoreduced set and let $f \in R\{Y\}$. Let $[G]$ denote the differential ideal generated by $G$ in $R\{Y\}$ and let $(G)$ denote the ideal generated by $G$ in $R\{Y\}$. Then there is some $\tilde{f} \in R\{Y\}$ which is reduced with respect to $G$ and some $H \in H_{G}$ such that $H \cdot f \equiv \tilde{f} \bmod [G]$. If $f$ is weakly reduced with respect to $G$, then we can take $H$ such that $H \cdot f \equiv \tilde{f} \bmod (G)$.

Proof. [Ko], Chap. I, Section 9, Proposition 1.

(2.10) Corollary. If $G$ is a characteristic set of a differential prime ideal $\mathfrak{p}$ of $R\{Y\}$ with $\mathfrak{p} \cap R=0$, then

$$
\mathfrak{p}=\left\{f \in R\{Y\} \mid H(G)^{n} \cdot f \in[G] \text { for some } n \in \mathbb{N}_{0}\right\} .
$$

Moreover if $f \in \mathfrak{p}$ is weakly reduced with respect to $G$, then $H(G)^{n} \cdot f \in(G)$ for some $n \in \mathbb{N}_{0}$.

Proof. From Theorem 2.9 and Proposition 2.7, since $H_{G} \cap \mathfrak{p}=\emptyset$.

Finally we collect some facts, which will be used later on.

(2.11) Proposition. Let $F \subseteq L$ be an extension of fields of characteristic 0 and let $F$ be equipped with $K$ commuting derivatives. Then there are $K$ commuting derivatives on $L$ extending those on $F$.

Proof. [Ko], p. 90.

(2.12) Proposition. Let $F$ be a differential field of characteristic 0 and let $Y$ be a set of differential indeterminates. Let $\mathfrak{a} \subseteq F\{Y\}$ be a differential ideal. Then:

(i) Every prime ideal $\mathfrak{p}$ of $F\{Y\}$, minimal with the property $\mathfrak{a} \subseteq \mathfrak{p}$, is a differential ideal.

(ii) Let $\mathfrak{a}$ be radical and differential. Then $\mathfrak{a}$ is finitely generated as a differential radical ideal. Moreover, if $F \subseteq L$ is an extension of differential fields, then the ideal generated by $\mathfrak{a}$ in $L\{Y\}$ is differential and radical.

Proof. For (i) see [Ko], Chap. 1, Sect. 2. Item (ii) can be found in [Ko], Chap. 4, Sect. 4. 
(2.13) Proposition. Let $F \subseteq L$ be fields of characteristic 0 and let $Y$ be a set of indeterminates. Let $G \subseteq F[Y]$ be a set and let $H \subseteq F[Y]$ be a multiplicatively closed set. Let $\mathfrak{a}$ be the ideal $(G)_{F[Y]}: H$ of $F[Y]$. Then the ideal $(G)_{L[Y]}: H$ of $L[Y]$ is generated by $\mathfrak{a}$ and $(G)_{L[Y]}: H \cap F[Y]=\mathfrak{a}$. Here, for an ideal $\mathfrak{a}$ of a ring $A$ and a subset $H$ of $A$, we write $\mathfrak{a}: H$ for the ideal $\{a \in A \mid h \cdot a \in \mathfrak{a}$ for some $h \in H\}$ of $A$.

Proof. We omit the easy proof.

(2.14) Theorem (The Rosenfeld Lemma). Let $F$ be a differential field of characteristic 0 in $K$ derivatives and let $A$ be the differential polynomial ring of $F$ in $Y:=\left(Y_{1}, \ldots, Y_{N}\right)$. Let $G \subseteq A$ be an autoreduced set. Then the following are equivalent:

1. $G$ is a characteristic set of $[G]: H(G)^{\infty}$ and $[G]: H(G)^{\infty}$ is prime.

2. (a) $G$ is coherent and

(b) the ideal $(G)_{A}: H(G)^{\infty}$ of $A$ is prime and does not contain nonzero elements of $A$, reduced with respect to $G$.

3. Let $B$ denote the $R$-algebra $F[y \in \mathcal{D} Y \mid y$ appears in $g$ for some $g \in G]$.

(a) $G$ is coherent and

(b) the ideal $(G)_{B}: H(G)^{\infty}$ of $B$ is prime and does not contain nonzero elements of $B$, reduced with respect to $G$.

Proof. $1 \Leftrightarrow 2$ is [ $\mathrm{Ko}$, IV, 9 , Lemma 2 , and $2 \Leftrightarrow 3$ can be easily derived.

Note. This significant characterization of a characteristic set can be generalized to the case of differential domains $R$ containing $\mathbb{Z}$ in $K$ commuting derivatives and certain differential radical ideals $\mathfrak{a}$ of $R\left\{Y_{1}, \ldots, Y_{N}\right\}$ with $\mathfrak{a} \cap R=\{0\}$. We will not make use of this and refer the reader to $[\mathrm{Hu}$.

(2.15) Proposition. Let $F \subseteq L$ be differential fields of characteristic 0 . If $G \subseteq$ $F\{Y\}$ is a characteristic set of $F\{Y\}$, then $G$ is a characteristic set of $L\{Y\}$, too.

Proof. Again, this is easy and left to the reader.

\section{Algebraically prepared systems}

(3.1) Definition. Let $F$ be a differential field of characteristic 0 in $K$ commuting derivatives. For every set $I \subseteq F\{Y\}$ of differential polynomials in the differential indeterminates $Y=\left(Y_{1}, \ldots, Y_{N}\right)$ we write

$$
A(I):=F\left[\Theta Y_{j} \mid \Theta \in \mathcal{D}, j \in\{1, \ldots, N\} \text { and } \Theta Y_{j} \text { appears in some } f \in I\right] .
$$

An algebraically prepared system of $F$ in $K$ derivatives is a sequence $\left(f_{1}, \ldots, f_{n}\right)$ of differential polynomials $f_{1}, \ldots, f_{n} \in F\left\{Y_{1}, \ldots, Y_{N}\right\} \backslash F$ such that the following two conditions hold:

(AP1) $\left\{f_{1}, \ldots, f_{n}\right\}$ is a characteristic set of a differential prime ideal, thus $\left\{f_{1}, \ldots, f_{n}\right\}$ is an autoreduced and coherent set of $n$ polynomials and the ideal $\left(f_{1}, \ldots, f_{n}\right): H\left(f_{1}, \ldots, f_{n}\right)^{\infty}$ of $A\left(f_{1}, \ldots, f_{n}\right)$ does not contain nonzero elements, reduced with respect to $f_{1}, \ldots, f_{n}$.

(AP2) The ideal $\left(f_{1}, \ldots, f_{n}\right): H\left(f_{1}, \ldots, f_{n}\right)^{\infty}$ of $A\left(f_{1}, \ldots, f_{n}\right)$ is prime and there is a regular $F$-rational point of this ideal, where $H\left(f_{1}, \ldots, f_{n}\right)$ does not vanish. 
We suppress the term "in $K$ derivatives" frequently, since $K$ will always be fixed. We say that $F$ solves an algebraically prepared system $\left(f_{1}, \ldots, f_{n}\right)$ if there is a differential solution $a \in F^{N}$ of $f_{1}=0, \ldots, f_{n}=0$.

We say that an algebraically prepared system $\left(f_{1}, \ldots, f_{n}\right)$ of $F$ is defined over a subring $R$ of $F$ if each $f_{i}$ is a polynomial over $R$.

(3.2) Lemma. Let $A$ be a domain, let $\mathfrak{a}$ be an ideal of $A$, let $h \in A$ and let $z$ be an indeterminate over $A$. Then

$$
\mathfrak{a}: h^{\infty}:=\left\{a \in A \mid h^{n} \cdot a \in \mathfrak{a} \text { for some } n \in \mathbb{N}\right\}=(\mathfrak{a}, z \cdot h-1)_{A[z]} \cap A .
$$

Moreover $h \notin \sqrt{\mathfrak{a}} \Longleftrightarrow A \neq \mathfrak{a}: h^{\infty}$, and in this case $h$ is a nonzero divisor of $A /\left(\mathfrak{a}: h^{\infty}\right)$. Also, the induced map $\left(A /\left(\mathfrak{a}: h^{\infty}\right)\right)_{h /\left(\mathfrak{a}: h^{\infty}\right)} \longrightarrow A[z] /(\mathfrak{a}, z \cdot h-1)$ which sends $\frac{1}{h /\left(\mathfrak{a}: h^{\infty}\right)}$ to $z /(\mathfrak{a}, z \cdot h-1)$ is an isomorphism. In particular, $\mathfrak{a}: h^{\infty}$ is prime if and only if $(\mathfrak{a}, z \cdot h-1)_{A[z]}$ is prime, provided that $h \notin \sqrt{\mathfrak{a}}$.

Proof. We omit the easy proof.

Notation. If $M, N$ are $\mathcal{L}$-structures in an arbitrary language and $A$ is a common subset of $M, N$, then we write

$$
M \equiv\rangle_{\exists, A} N
$$

if every existential $\mathcal{L}$-formula with parameters in $A$, that holds in $M$, also holds in $N$. We write $M \equiv_{\exists, A} N$ if $M \equiv_{\exists, A} N$ and $\left.N \equiv\right\rangle_{\exists, A} M$. Hence $M \equiv_{\exists, A} N$ if and only if $M$ and $N$ have the same universal theory over $A$ as explained in the Introduction.

(3.3) Theorem. Let $A$ be a common differential subring of differential fields $L_{1}, L_{2}$ of characteristic 0 in $K$ commuting derivatives. Let $F_{i}$ be the algebraic closure of the quotient field $F_{0}$ of $A$ in $L_{i}$. Then:

(i) $L_{1} \equiv_{\exists, A} L_{2}$ as pure fields in the language of rings and

(ii) $L_{2}$ solves all algebraically-prepared systems of $L_{2}$, defined over $F_{2}$.

Then $\left.L_{1} \equiv\right\rangle_{\exists, F_{0}} L_{2}$ as differential fields in the language of differential rings in $K$ derivatives.

Proof. By a standard argument, condition (i) implies $L_{1} \equiv_{\exists, F_{0}} L_{2}$ as pure fields and that $F_{1}$ and $F_{2}$ are isomorphic as fields over $F_{0}$. This isomorphism respects the derivatives, too (observe that $F_{i}$ is a differential subfield of $L_{i}$ ). Hence we may assume that $F:=F_{1}=F_{2}$ is the algebraic closure of $F_{0}=A$ in $L_{i}$.

Let $\varphi(\bar{x})$ be a quantifier-free formula in the language of differential rings, with parameters from $F_{0}$, where $\bar{x}$ is an $N$-tuple of variables. Suppose there is some $\bar{a} \in L_{1}^{N}$ such that $L_{1} \models \varphi(\bar{a})$. We have to find some $\bar{b} \in L_{2}^{N}$ such that $L_{2} \models \varphi(\bar{b})$. Clearly we may assume that $\varphi(\bar{x})$ is of the form $p_{1}(\bar{x})=0 \wedge \ldots \wedge p_{r}(\bar{x})=0 \wedge q(\bar{x}) \neq 0$ with differential polynomials $p_{1}, \ldots, p_{r}, q \in F_{0}\{Y\}$, where $Y:=\left(Y_{1}, \ldots, Y_{N}\right)$. By replacing $q(\bar{x}) \neq 0$ with $x_{n+1} \cdot q(\bar{x})-1=0$ if necessary we may assume that $\varphi(\bar{x})$ is of the form $p_{1}(\bar{x})=0 \wedge \ldots \wedge p_{r}(\bar{x})=0$.

Let $\mathfrak{p}_{1}:=\{f \in F\{Y\} \mid f(\bar{a})=0\}$ and let $\mathfrak{p}_{0}:=\left\{f \in F_{0}\{Y\} \mid f(\bar{a})=0\right\}$. Then $\mathfrak{p}_{1}$ and $\mathfrak{p}_{0}$ are differential prime ideals of $F\{Y\}$ and $F_{0}\{Y\}$, respectively. Moreover $\mathfrak{p}_{1}$ lies over $\mathfrak{p}_{0}$ and $p_{1}, \ldots, p_{r} \in \mathfrak{p}_{0}$. Let $f_{1}, \ldots, f_{n}$ be a characteristic set of $\mathfrak{p}_{1}$. As $F$ is algebraic over $F_{0}$, there is some $\alpha \in F$ such that the polynomials $f_{1}, \ldots, f_{n}$ and $g$ have coefficients in $F_{0}(\alpha)$. Since $F\{Y\}$ is a differentially noetherian ring and $F$ is algebraic over $F_{0}$, there are only finitely many differential prime ideals of $F\{Y\}$ 
lying over $\mathfrak{p}_{0}$; say there are exactly $s$ of them. Since $F$ is algebraic over $F_{0}$ we may choose $\alpha$ so that in addition, there are $s$ differential prime ideals of $F_{0}(\alpha)\{Y\}$ lying over $\mathfrak{p}_{0}$.

Let $\bar{u}=\left(u_{1}, \ldots, u_{r}\right)$ be an enumeration of all variables $\Theta Y_{j}$ occurring in some of the $f_{1}, \ldots, f_{n}$. We write $f_{i}(\bar{u})$ for $f_{i}(Y)$ when $f_{i}$ is viewed as an ordinary polynomial in $\bar{u}$. For $i \in\{1, \ldots, r\}$ let $c_{i}:=\Theta a_{j}$ if $u_{i}=\Theta Y_{j}$ and let $\bar{c}:=\left(c_{1}, \ldots, c_{r}\right)$.

By definition of $\mathfrak{p}_{1}, \bar{c}$ is a generic solution of the ideal $\left(f_{1}, \ldots, f_{n}\right): H\left(f_{1}, \ldots, f_{n}\right)^{\infty}$ of $A\left(f_{1}, \ldots, f_{n}\right) \subseteq F\{Y\}$ (note that $\left.\left(f_{1}, \ldots, f_{n}\right): H\left(f_{1}, \ldots, f_{n}\right)^{\infty}=\mathfrak{p}_{1} \cap A\left(f_{1}, \ldots, f_{n}\right)\right)$. Hence $\bar{c}$ is a regular point of the ideal $\left(f_{1}, \ldots, f_{n}\right): H\left(f_{1}, \ldots, f_{n}\right)^{\infty}$ and $H\left(f_{1}, \ldots, f_{n}\right)(\bar{c})$ $\neq 0$. By $3.2\left(\bar{c}, 1 / H\left(f_{1}, \ldots, f_{n}\right)(\bar{c})\right)$ is a regular point of the prime ideal $\left(f_{1}, \ldots, f_{n}, u_{0}\right.$. $\left.H\left(f_{1}, \ldots, f_{n}\right)-1\right)$ of $A\left(f_{1}, \ldots, f_{n}\right)\left[u_{0}\right]$, where $u_{0}$ is a new indeterminate. Hence the jacobian of $\left(f_{1}, \ldots, f_{n}, u_{0} \cdot H\left(f_{1}, \ldots, f_{n}\right)-1\right)$ has rank $r+1-d$, where $d$ is the Krull dimension of $\left(f_{1}, \ldots, f_{n}\right): H\left(f_{1}, \ldots, f_{n}\right)^{\infty}$ (which is the same as the Krull dimension of $\left.\left(f_{1}, \ldots, f_{n}, u_{0} \cdot H\left(f_{1}, \ldots, f_{n}\right)-1\right)\right)$. Take $f_{0}:=u_{0} \cdot H\left(f_{1}, \ldots, f_{n}\right)-1$ and let $i_{1}<\ldots<i_{r+1-d} \in\{0, \ldots, r\}, j_{1}<\ldots<j_{r+1-d} \in\{0, \ldots, n\}$ such that the determinant of $\left(\frac{\partial}{\partial u_{i_{k}}} f_{j_{m}}\right)_{k, m \in\{1, \ldots, r+1-d\}}$ does not vanish at $\bar{c}$.

Let $Z$ be a new differential indeterminate of rank smaller than $Y_{1}, \ldots, Y_{n}$, and let $\mu(Z) \in F[Z]$ be the minimal polynomial of $\alpha$ over $F_{0}$. For $\bar{\varepsilon} \in \mathbb{N}_{0}^{r}$ and $i \in\{1, \ldots, n\}$ let $f_{i, \varepsilon} \in F_{0}[Z]$ be the uniquely determined polynomials of degree $<\left[F_{0}(\alpha): F_{0}\right]$ such that $f_{i}(\bar{u})=f_{i}^{*}(\bar{u}, \alpha)$, where

$$
f_{i}^{*}(\bar{u}, Z):=\sum_{\varepsilon \in \mathbb{N}_{0}^{r}} f_{i, \varepsilon}(Z) \bar{u}^{\varepsilon} .
$$

Again we write $f_{i}^{*}(Y, Z)$ if we consider $f_{i}^{*}(\bar{u}, Z)$ as a differential polynomial in $Z, Y_{1}, \ldots, Y_{N}$.

Then for all zeroes $\gamma$ of $\mu$ we have

$$
H\left(f_{1}^{*}(Y, Z), \ldots, f_{n}^{*}(Y, Z)\right)(Y, \gamma)=H\left(f_{1}^{*}(Y, \gamma), \ldots, f_{n}^{*}(Y, \gamma)\right) \text {, }
$$

thus with $f_{0}^{*}:=u_{0} \cdot H\left(f_{1}^{*}(Y, Z), \ldots, f_{n}^{*}(Y, Z)\right)-1$ we get

$$
\left(\frac{\partial}{\partial u_{j}} f_{i}^{*}\right)(\bar{u}, \gamma)=\frac{\partial}{\partial u_{j}}\left(f_{i}^{*}(\bar{u}, \gamma)\right)(0 \leq i \leq n, 0 \leq j \leq r) .
$$

Since $\left.L_{1} \equiv\right\rangle_{\exists, F_{0}} L_{2}$ as rings, there is a solution of the ordinary polynomial system

$$
\begin{gathered}
f_{1}^{*}(\bar{u}, Z)=0, \ldots, f_{n}^{*}(\bar{u}, Z)=0, u_{0} \cdot H\left(f_{1}^{*}, \ldots, f_{n}^{*}\right)(\bar{u}, Z)-1=0, \mu(Z)=0, \\
\operatorname{det}\left(\frac{\partial}{\partial u_{i_{k}}} f_{j_{m}}^{*}\right)_{k, m \in\{1, \ldots, r+1-d\}} \neq 0 \text { in } L_{2} .
\end{gathered}
$$

In other words, there is a zero $\beta$ of $\mu(Z)$ in $L_{2}$ such that the ideal

$$
\left(f_{1}^{*}(\bar{u}, \beta), \ldots, f_{n}^{*}(\bar{u}, \beta), u_{0} \cdot H\left(f_{1}^{*}, \ldots, f_{n}^{*}\right)(\bar{u}, \beta)-1\right)
$$

of $F_{0}(\beta)\left[u_{0}, \bar{u}\right]$ has a solution $\bar{d}$ in $L_{2}^{r+1}$ with the property

$$
\operatorname{det}\left(\frac{\partial}{\partial u_{i_{k}}} f_{j_{m}}^{*}(\bar{u}, \beta)\right)_{k, m \in\{1, \ldots, r+1-d\}} \neq 0 \text { at } \bar{d}
$$

Let $\sigma: F_{0}(\alpha) \longrightarrow F_{0}(\beta)$ be the $F_{0}$-isomorphism sending $\alpha$ to $\beta$. We also write $\sigma$ for the canonical extension of $\sigma$ to the differential polynomial rings $F_{0}(\alpha)\{Y\} \longrightarrow$ $F_{0}(\beta)\{Y\}$. Then $\sigma\left(f_{1}\right)=f_{1}^{*}(Y, \beta), \ldots, \sigma\left(f_{n}\right)=f_{n}^{*}(Y, \beta)$. Since $\sigma$ is a differential $F_{0}\{Y\}$-isomorphism $F_{0}(\alpha)\{Y\} \longrightarrow F_{0}(\beta)\{Y\}$ and $\left\{f_{1}, \ldots, f_{n}\right\}$ is a characteristic set of $\mathfrak{p}_{1} \cap F_{0}(\alpha)\{Y\}$, the sequence $\left(\sigma\left(f_{1}\right), \ldots, \sigma\left(f_{n}\right)\right)$ is a characteristic set of $\sigma\left(\mathfrak{p}_{1} \cap F_{0}(\alpha)\{Y\}\right)$. 
Claim. $\left(\sigma\left(f_{1}\right), \ldots, \sigma\left(f_{n}\right)\right)$ is an algebraically prepared system of $L_{2}$.

As $\sigma\left(f_{1}\right), \ldots, \sigma\left(f_{n}\right)$ is a characteristic set of $\sigma\left(\mathfrak{p}_{1} \cap F_{0}(\alpha)\{Y\}\right), \sigma\left(f_{1}\right), \ldots ., \sigma\left(f_{n}\right)$ is autoreduced and coherent. These conditions are not violated if we enlarge the coefficients (cf. 2.15). Hence $\left(\sigma\left(f_{1}\right), \ldots, \sigma\left(f_{n}\right)\right)$ is a characteristic set of $F\{Y\}$ and $L_{2}\{Y\}$. We now show that the ideal $\left(\sigma\left(f_{1}\right), \ldots, \sigma\left(f_{n}\right)\right): H\left(\sigma\left(f_{1}\right), \ldots, \sigma\left(f_{n}\right)\right)^{\infty}$ of $L_{2}[\bar{u}]$ is prime. By 2.13 this ideal is generated by the ideal

$$
\mathfrak{a}_{0}:=\left(\sigma\left(f_{1}\right), \ldots, \sigma\left(f_{n}\right)\right): H\left(\sigma\left(f_{1}\right), \ldots, \sigma\left(f_{n}\right)\right)^{\infty}
$$

of $F[\bar{u}]$. As $F$ is algebraically closed in $L_{2}$ it is enough to show that $\mathfrak{a}_{0}$ is prime. By 2.14, it is enough to show that the differential ideal $\mathfrak{a}:=\left[\sigma\left(f_{1}\right), \ldots, \sigma\left(f_{n}\right)\right]$ : $H\left(\sigma\left(f_{1}\right), \ldots, \sigma\left(f_{n}\right)\right)^{\infty}$ of $F\{Y\}$ is prime. By 2.13 this ideal is generated by the differential ideal $\left[\sigma\left(f_{1}\right), \ldots, \sigma\left(f_{n}\right)\right]: H\left(\sigma\left(f_{1}\right), \ldots, \sigma\left(f_{n}\right)\right)^{\infty}$ of $F_{0}(\beta)\{Y\}$, which is equal to $\sigma\left(\mathfrak{p}_{1} \cap F_{0}(\alpha)\{Y\}\right)$. Now $\mathfrak{a}$ is radical and the claim follows if we know that there is a unique differential prime ideal of $F\{Y\}$ lying over $\sigma\left(\mathfrak{p}_{1} \cap F_{0}(\alpha)\{Y\}\right)$ (cf. 2.12). The isomorphism $\sigma$ maps the set of differential prime ideals of $F_{0}(\alpha)\{Y\}$ lying over $\mathfrak{p}_{0}$ bijectively onto the set of differential prime ideals of $F_{0}(\beta)\{Y\}$ lying over $\mathfrak{p}_{0}$. Hence by our choice of $\alpha$, there are $s$ differential prime ideals of $F_{0}(\beta)\{Y\}$ lying over $\mathfrak{p}_{0}$. By choice of $s$ there are $s$ differential prime ideals of $F\{Y\}$ lying over $\mathfrak{p}_{0}$. Hence there can only be one differential prime ideal of $F\{Y\}$ lying over $\sigma\left(\mathfrak{p}_{1} \cap F_{0}(\alpha)\{Y\}\right)$. This shows that the ideal $\left(\sigma\left(f_{1}\right), \ldots, \sigma\left(f_{n}\right)\right): H\left(\sigma\left(f_{1}\right), \ldots, \sigma\left(f_{n}\right)\right)^{\infty}$ of $L_{2}[\bar{u}]$ is prime.

By 3.2, the ideal $\left(f_{1}^{*}(\bar{u}, \beta), \ldots, f_{n}^{*}(\bar{u}, \beta), u_{0} \cdot H\left(f_{1}^{*}, \ldots, f_{n}^{*}\right)(\bar{u}, \beta)-1\right)$ of $L_{2}\left[u_{0}, \bar{u}\right]$ is prime and condition (*) above says that $\bar{d}$ is a regular solution of this ideal. Again, this means that $\bar{d}$ is a regular solution of the prime ideal $\left(\sigma\left(f_{1}\right), \ldots, \sigma\left(f_{n}\right)\right)$ : $H\left(\sigma\left(f_{1}\right), \ldots, \sigma\left(f_{n}\right)\right)^{\infty}$ of $L_{2}[\bar{u}]$, where $H\left(\sigma\left(f_{1}\right), \ldots, \sigma\left(f_{n}\right)\right)$ does not vanish. This finishes the proof of the claim.

By the claim and our assumption (ii) (observe that the algebraically prepared system $\left(\sigma\left(f_{1}\right), \ldots, \sigma\left(f_{n}\right)\right)$ of $L_{2}$ is defined over $\left.F\right)$ we get a differential solution $\bar{b} \in L_{2}^{N}$ of $\sigma\left(f_{1}\right)=0, \ldots, \sigma\left(f_{n}\right)=0$. As $p_{1}, \ldots, p_{r} \in \mathfrak{p}_{0}$ and

$$
\sigma\left(\mathfrak{p}_{1} \cap F_{0}(\alpha)\{Y\}\right)=\left[\sigma\left(f_{1}\right), \ldots, \sigma\left(f_{n}\right)\right]: H\left(\sigma\left(f_{1}\right), \ldots, \sigma\left(f_{n}\right)\right)^{\infty}
$$

lies over $\mathfrak{p}_{0}, \bar{b}$ is also a differential solution of $p_{1}=\ldots=p_{r}=0$, which means $L_{2} \models \varphi(\bar{b})$.

This is a good point to describe how our theory $\mathrm{UC}_{K}$ will be axiomatized. The theory $\mathrm{UC}_{K}$, yet to be defined, will say the following about a model $F$ :

"every algebraically prepared system in $K$ derivatives, defined over $F$ has a differential solution in $F "$.

By 3.3 such a theory will have property (I) stated in the Introduction. So what remains to do is to axiomatize the sentence "every algebraically prepared system defined over $F$ has a differential solution in $F$ " and to prove property (II) stated in the Introduction.

\section{Definition of UC}

Again we fix $K \in \mathbb{N}$, the number of derivatives. We shall show:

(4.1) Proposition. Let $n, N \in \mathbb{N}$, let $Y=\left(Y_{1}, \ldots, Y_{N}\right)$ and let $f_{1}(\bar{t}, Y), \ldots, f_{n}(\bar{t}, Y)$ $\in \mathbb{Z}\{Y\}[\bar{t}]$ be general polynomials in the indeterminates $\left\{\Theta Y_{j} \mid \Theta \in \mathcal{D}, 1 \leq j \leq N\right\}$, in the indeterminate coefficients $\bar{t}=\left(t_{1}, \ldots, t_{r}\right)$. Then there is a formula $\varphi(\bar{v})$, 
$\bar{v}=\left(v_{1}, \ldots, v_{r}\right)$, such that for all differential fields $F$ of characteristic 0 in $K$ commuting derivatives and all $\bar{c} \in F^{r}$ we have

$F \models \varphi(\bar{c}) \Longleftrightarrow\left(f_{1}(\bar{c}, Y), \ldots, f_{n}(\bar{c}, Y)\right)$ is an algebraically prepared system of $F$.

The proof of 4.1 divides into two parts: we will show that condition (AP1) and condition (AP2) of 3.1 are definable conditions on the coefficients of a sequence of differential polynomials. First we recall some facts from the paper vdD-Sch.

(4.2) Theorem. Let $n, d \in \mathbb{N}, X=\left(X_{1}, \ldots, X_{n}\right)$. Then there are bounds $B=$ $B(n, d), C=C(n, d)$ and $E=E(n, d)$ in $\mathbb{N}$ such that for each field $F$, each ideal $I$ of $K[X]$ generated by polynomials of degree $\leq d$ and all $f_{1}, \ldots, f_{l} \in F[X]$ of degree $\leq d$ the following are true:

(i) If $I$ is generated by $f_{1}, \ldots, f_{l}$ and each of the $f_{i}$ is of degree $\leq d$, then for every polynomial $g \in I$ of degree $\leq d$, there are polynomials $c_{1}, \ldots, c_{l} \in F[X]$ of degree $\leq E(n, d)$ such that $g=c_{1} \cdot f_{1}+\ldots+c_{l} \cdot f_{l}$.

(ii) $I$ is prime $\Longleftrightarrow 1 \notin I$, and for all $f, g \in F[X]$ of degree $\leq B$, if $f \cdot g \in I$, then $f \in I$ or $g \in I$.

(iii) For all $m \in\{1, \ldots, n\}$ the ideal $I \cap F\left[X_{1}, \ldots, X_{m}\right]$ is generated by at most $C(n, d)$ polynomials of degree $\leq C(n, d)$.

Proof. (i) is a special case of vdD-Sch, Thm. (1.11), (ii) is a part of vdD-Sch, Thm. (2.10), and (iii) is a basic statement in the theory of Gröbner Bases (e.g., [E], Prop. 15.29).

(4.3) Lemma. Let $n, N \in \mathbb{N}$, let $Y=\left(Y_{1}, \ldots, Y_{N}\right)$ and let $f_{1}(\bar{t}, Y), \ldots, f_{n}(\bar{t}, Y) \in$ $\mathbb{Z}\left[\bar{t}\{Y\}\right.$ be general polynomials in the indeterminates $\left\{\Theta Y_{j} \mid \Theta \in \mathcal{D}, 1 \leq j \leq N\right\}$, with indeterminate coefficients $\bar{t}=\left(t_{1}, \ldots, t_{r}\right)$. Then there is a formula $\varphi_{2}(\bar{v}), \bar{v}=$ $\left(v_{1}, \ldots, v_{r}\right)$, such that for all differential fields $F$ of characteristic 0 in $K$ commuting derivatives and all $\bar{c} \in F^{r}$ we have $F \models \varphi_{2}(\bar{c})$ if and only if the ideal $\left(f_{1}, \ldots, f_{n}\right)$ : $H\left(f_{1}, \ldots, f_{n}\right)^{\infty}$ of $A\left(f_{1}, \ldots, f_{n}\right)$ is prime and there is a regular $F$-rational point of this ideal, where $H\left(f_{1}, \ldots, f_{n}\right)$ does not vanish.

Recall that $A\left(f_{1}, \ldots, f_{n}\right)$ is the ordinary polynomial ring over $F$ in those variables $\Theta Y_{j}$ which appear in some of the $f_{i}$.

Proof. By 3.2 the condition in question is equivalent to the condition "The ideal $\mathfrak{a}:=\left(f_{1}, \ldots, f_{n}, u_{0} \cdot H\left(f_{1}, \ldots, f_{n}\right)-1\right)$ of $A\left(f_{1}, \ldots, f_{n}\right)\left[u_{0}\right]$ is prime and there is a regular $F$-rational point of this ideal". Here $u_{0}$ is a new variable. The primality of this ideal is definable by 4.2(ii). The existence of a regular $F$-rational point of this ideal can be expressed in a first-order formula with the Jacobian criterion; for this, also observe that the Krull dimension of the prime ideal $\mathfrak{a}$ is a definable expression in terms of the coefficients.

In order to formulate (AP1) of 3.2 in a first order way we use

(4.4) Theorem. Let $F$ be a field, let $X_{1}, \ldots, X_{n}$ be indeterminates over $F$ and let $s, d \in \mathbb{N}$. Then there is a natural number $B(n, d, s)$ such that whenever $f_{1}, \ldots, f_{s} \in$ $F\left[X_{1}, \ldots, X_{n}\right]$ are polynomials of total degree $\leq d$, then the ideal $\left(f_{1}, \ldots, f_{s}\right)$ has a characteristic set $\left\{g_{1}, \ldots, g_{r}\right\}$ for some $r \leq n$ such that the total degree of each $g_{j}$ is at most $B(n, d, s)$.

Proof. This is GaMi], Theorem 3.4, applied to the ordinary polynomial ring over $F$ in the variables occurring in one of the $f_{i}$. Note that in this paper, a characteristic set of this ideal is defined exactly in the same way as we do in 2.6. $\mathrm{cf}$. 
GaMi], Definition 2.6. Note also that in this paper, a bound $B(n, d, s)$ is explicitly calculated.

Now we can prove 4.1, By 2.14 a sequence $\left(f_{1}, \ldots, f_{n}\right) \in F\{Y\}$ with the property that $\left(f_{1}, \ldots, f_{n}\right): H\left(f_{1}, \ldots, f_{n}\right)^{\infty}$ is a prime ideal of $A\left(f_{1}, \ldots, f_{n}\right)$, is a characteristic set of a differential prime ideal of $F\{Y\}$ if and only if it is a characteristic set of the ideal $\left(f_{1}, \ldots, f_{n}\right): H\left(f_{1}, \ldots, f_{n}\right)^{\infty}$ of $A\left(f_{1}, \ldots, f_{n}\right)$ and if it is an autoreduced and coherent set of differential polynomials. The reducedness and the coherence are certainly definable in terms of the coefficients of the $f_{1}, \ldots, f_{n}$. The first condition, that $\left(f_{1}, \ldots, f_{n}\right)$ is a characteristic set of $\left(f_{1}, \ldots, f_{n}\right): H\left(f_{1}, \ldots, f_{n}\right)^{\infty} \subseteq A\left(f_{1}, \ldots, f_{n}\right)$, is definable in terms of the coefficients of $f_{1}, \ldots, f_{n}$ by [3.2, 4.2 and 4.4. By 3.2 and 4.2(iii), there is a natural number $E(d, r)$ only depending on the degree $d$ of the $f_{1}, \ldots, f_{n}$ and the number $r$ of ordinary variables in $A\left(f_{1}, \ldots, f_{n}\right)$ such that the ideal $\left(f_{1}, \ldots, f_{n}\right): H\left(f_{1}, \ldots, f_{n}\right)^{\infty}$ of $A\left(f_{1}, \ldots, f_{n}\right)$ is generated by at most $E(d, r)$ polynomials of degree $\leq E(d, r)$. By applying 4.4 to this ideal and the bound $E(d, r)$ we get a bound $B(d, r)$ only depending on $d$ and $r$ such that the ideal $\left(f_{1}, \ldots, f_{n}\right): H\left(f_{1}, \ldots, f_{n}\right)^{\infty}$ has a characteristic set $g_{1}, \ldots, g_{l}$ for some $l \leq E(d, r)$ where all $g_{i}$ are of degree $\leq B(d, r)$. By 4.2 (i) it follows that we can express the statement " $\left(f_{1}, \ldots, f_{n}\right)$ is a characteristic set of $\left(f_{1}, \ldots, f_{n}\right): H\left(f_{1}, \ldots, f_{n}\right)^{\infty}$ " in a first order way, in terms of the coefficients: we say that no system of at most $E(d, r)$ polynomials from $\left(f_{1}, \ldots, f_{n}\right): H\left(f_{1}, \ldots, f_{n}\right)^{\infty}$ has a rank strictly less than the rank of $\left(f_{1}, \ldots, f_{n}\right)$.

Together with 4.3 this gives a formula $\varphi_{1}(\bar{v})$, where $\bar{v}=\left(v_{1}, \ldots, v_{r}\right)$ and $r$ is the length of the coefficient vector of $\left(f_{1}, \ldots, f_{n}\right)$, such that for all differential fields $F$ of characteristic 0 in $K$ commuting derivatives and all $\bar{c} \in F^{r}$, we have $F \models \varphi_{2}(\bar{c})$ if and only if $\left(f_{1}, \ldots, f_{n}\right)$ is a characteristic set of a differential prime ideal of $F\{Y\}$.

Together with 4.3 this completes the proof of 4.1 take $\varphi(\bar{v})=\varphi_{1}(\bar{v}) \wedge \varphi_{2}(\bar{v})$.

(4.5) Corollary and Definition. Let $K \in \mathbb{N}$ and let $\mathcal{L}$ be the language $\{+,-, \cdot, 0,1\}$ of rings. The class of all differential fields of characteristic 0 in $K$ commuting derivatives, which solve all their algebraically prepared systems, is axiomatizable in the language $\mathcal{L}\left(\partial_{1}, \ldots, \partial_{K}\right)$. The theory of this class is denoted by $\mathrm{UC}$ (for Uniform Companion). If we want to point out the number $K$ of derivatives involved, we write $\mathrm{UC}_{K}$.

Proof. By 4.1.

Hence UC is defined and by 3.3 we know that property (I), stated in the Introduction, holds. Before proving assertion (II) from the Introduction, we first recall some facts about large fields.

\section{LARGE FIELDS}

(5.1) Definition. A field $F$ is called large if every smooth integral curve defined over $F$ that has an $F$-rational point has infinitely many $F$-rational points (cf. $[\overline{\mathrm{Po}}$ ). Let $T$ be a theory of fields in a language extending the language of rings. We say that $T$ is large if all models of $T$ are large fields.

(5.2) Lemma. The class of all large fields is axiomatizable in the language of rings.

Proof. Let $f_{1}, \ldots, f_{n}(\bar{U}, \bar{X}) \in \mathbb{Z}[\bar{U}, \bar{X}]$. There is a formula $\varphi(\bar{u})$ in the language of rings, such that for every field $F$ and all $\bar{U}$-tuples $\bar{c}$ from $F, F \models \varphi(\bar{c})$ if and only 
if the system $f_{1}(\bar{c}, \bar{X})=0, \ldots, f_{n}(\bar{c}, \bar{X})=0$ defines an integral, smooth curve over $F$, which has an $F$-rational point.

Therefore the set of all formulas

$$
\forall \bar{u} \varphi(\bar{u}) \rightarrow \exists \bar{x}_{1}, \ldots, \bar{x}_{n} \bigwedge_{i \neq j} \bar{x}_{i} \neq \bar{x}_{j} \wedge \bigwedge_{i} f_{1}\left(\bar{u}, \bar{x}_{i}\right)=0, \ldots, f_{n}\left(\bar{u}, \bar{x}_{i}\right)=0,
$$

where $n \in \mathbb{N}$, is equivalent to the statement that every smooth integral curve defined over $F$ by a system $f_{1}(\bar{c}, \bar{X})=0, \ldots, f_{n}(\bar{c}, \bar{X})=0, \bar{c} \subseteq F$, which has an $F$-rational point, has infinitely many $F$-rational points.

This also shows the lemma.

(5.3) Proposition. Let $F$ be a field of characteristic 0. The following are equivalent:

(i) $F$ is large.

(ii) $F$ is existentially closed in the formal Laurent series field $F((t))$ in one variable.

(iii) For all $n \in \mathbb{N}, F$ is existentially closed in the formal Laurent series field $F\left(\left(t_{1}, \ldots, t_{n}\right)\right)$.

(iv) For every $n \in \mathbb{N}$ and every prime ideal $\mathfrak{p} \subseteq F[X], X=\left(X_{1}, \ldots, X_{n}\right)$, if $V(\mathfrak{p}):=($ the zeroes of $\mathfrak{p}$ in the algebraic closure of $F$ ) has a regular, $F$ rational point, then $F$ is existentially closed in $F[X] / \mathfrak{p}$ (i.e. the $F$-rational points of $V(\mathfrak{p})$ are Zariski dense in $V(\mathfrak{p}))$.

Proof. (i) $\Rightarrow$ (ii). It is enough to show that $F$ is existentially closed in $F[[t]]$. Let $A$ be the ring of all elements of $F[[t]]$ which are algebraic over $F[t]$. By Artin approximation, $A$ is existentially closed in $F[[t]]$, and it is enough to show that $F$ is existentially closed in every finitely-generated $F$-algebra $A_{0} \subseteq A$. Since $A_{0}$ is integrally closed, the integral closure $B$ of $A_{0}$ is in $A$. Moreover, $B$ is finitely generated over $F$ and of dimension 1. Thus $B$ is a regular ring. Let $C$ be the curve defined by $B$ over $F$. Then $C$ is smooth and since $B \subseteq F[[t]], C$ has an $F$-rational point. Since $F$ is large, $C$ has infinitely many $F$-rational points which means that $F$ is existentially closed in $B$ as desired. This proves (i) $\Rightarrow$ (ii).

Now we prove for a given $d \in \mathbb{N}$ : if $F$ is existentially closed in $F\left[\left[t_{1}, \ldots, t_{d}\right]\right]$ and $\mathfrak{p} \subseteq F[X], X=\left(X_{1}, \ldots, X_{n}\right), n \in \mathbb{N}$, is a prime ideal of dimension $d$ such that $V(\mathfrak{p})$ has a regular, $F$-rational point, then $F$ is existentially closed in $F[X] / \mathfrak{p}$. This will show (ii) $\Rightarrow$ (i) and (iii) $\Rightarrow$ (iv).

In order to see the Claim, let $A:=F[X] / \mathfrak{p}$ and let $\bar{a}$ be an $F$-rational regular point of $V(\mathfrak{p})$. This means that the maximal ideal $\mathfrak{m}$ of $A$ corresponding to $\bar{a}$ has residue field $F$. Let $B$ be the $\mathfrak{m}$-adic completion of $A_{\mathfrak{m}}$. Since $\bar{a}$ is a regular point of $V(\mathfrak{p}), A_{\mathfrak{m}}$ is regular, local with residue field $F$, and $\operatorname{dim} A_{\mathfrak{m}}=d$. Then $A_{\mathfrak{m}} \subseteq B$ and $B$ is regular, complete, and local with residue field $F$. So $B$ is $F$-isomorphic to $F\left[\left[T_{1}, \ldots, T_{d}\right]\right]$.

This shows that $A$ can be embedded over $F$ into $F\left[\left[T_{1}, \ldots, T_{d}\right]\right]$. By assumption, $F$ is existentially closed in $F\left[\left[T_{1}, \ldots, T_{d}\right]\right]$; in particular $F$ is existentially closed in A.

Hence we know (i) $\Leftrightarrow($ ii) and (iii) $\Rightarrow($ iv). Since (i) is a weakening of (iv) it remains to show (ii) $\Rightarrow$ (iii). We show by induction on $n$ that $F$ is existentially closed in $F\left[\left[t_{1}, \ldots, t_{n}\right]\right]$, where $n=1$ holds by assumption. Assume we know that $F$ is existentially closed in $F\left[\left[t_{1}, \ldots, t_{n}\right]\right]$. Let $L \succ F$ such that there is an $F$-embedding $F\left[\left[T_{1}, \ldots, T_{d}\right]\right] \longrightarrow L$. Since $F$ is large, $L$ is large, too (by 5.2 and since we already 
know that (i) $\Leftrightarrow($ ii) $)$. Hence $L$ is existentially closed in $L\left[\left[T_{d+1}\right]\right]$, so $F$ is existentially closed in $\left.F\left[\left[T_{1}, \ldots ., T_{d+1}\right]\right] \subseteq L\left[\left[T_{d+1}\right]\right]\right)$.

Examples of large fields. All $P A C, P R C$ and all $P p C$-fields are large. Moreover, if $F$ is a field admitting a nontrivial henselian valuation, then $F$ is large (in section 8 we discuss this in more detail).

\section{Proof of the Main theorem}

We have to show that every differential field $F$ which is large as a pure field has an extension which is a model of UC and which is an elementary extension when considered as a pure field. This reduces to the problem of finding a differential solution of a given algebraically prepared system $\left(f_{1}, \ldots, f_{n}\right)$ of $F$ in a differential field $L \supseteq F$ such that $F$ is existentially closed in $L$ as a pure field. The following theorem translates this plan into a problem about finitely generated $F$-algebras.

(6.1) Theorem. Let $F$ be a differential field of characteristic 0 in $K$ commuting derivatives and let $\mathfrak{p} \subseteq F\{Y\}, Y:=\left(Y_{1}, \ldots, Y_{N}\right)$ be a differential prime ideal. Let $\varphi: F\{Y\} \longrightarrow F\{Y\} / \mathfrak{p}=: S$ be the residue map and let $G$ be a characteristic set of $\mathfrak{p}$. Let $H(G)$ be the product of all initials and separants of polynomials in $G$. Let $h:=\varphi(H(G))$,

$V:=\{y \in \mathcal{D} Y \mid y$ is not a proper derivative of any leader of an element $g \in G\}$,

$$
\begin{aligned}
V_{B} & :=\{y \in V \mid y \text { appeares in some } g \in G\}, \\
B & :=\varphi\left(F\left[V_{B}\right]\right) \text { and } P:=\varphi\left(F\left[V \backslash V_{B}\right]\right) .
\end{aligned}
$$

Then $h \in B, h \neq 0$ and

(a) $B$ is a finitely generated $R$-algebra and $P$ is $F$-isomorphic to a polynomial ring over $F$ in at most countably many variables (the case $P=F$ is not excluded).

(b) $S_{h}=(B \cdot P)_{h}$ is a differentially finitely generated $F$-algebra.

(c) The homomorphism $B \otimes_{F} P \longrightarrow B \cdot P$ induced from multiplication is an isomorphism of $F$-algebras.

(d) The restriction of $\varphi$ to $F\left[V \backslash V_{B}\right]$ is injective.

Proof. By $\operatorname{Tr}$; the theorem is stated there without the definition of the data $B, P$ and $h$ in the case where $F$ is a differential domain. The definition of the data can be found at the beginning of section $3 \mathrm{in}[\mathrm{Tr}$. Also, item (d) is claim 1 of section 3 in $\operatorname{Tr}$.

(6.2) Main Theorem. Let $K \in \mathbb{N}$. Then:

(I) Whenever $L$ and $M$ are models of $\mathrm{UC}_{K}$ and $A$ is a common differential subring of $L$ and $M$ such that $L$ and $M$ have the same universal theory over $A$ as pure fields, then they have the same universal theory over $A$ as differential fields.

(II) Every differential field $F$ which is large as a pure field can be extended to a model of $\mathrm{UC}_{K}$, and this extension is elementary in the language of rings.

Proof. (I) holds by 3.3. We prove (II).

Claim. If $F$ is a differential field, which is large as a pure field, and $\left(f_{1}, \ldots, f_{n}\right)$ is an algebraically prepared system of differential polynomials from $F\{Y\}$, $Y=\left(Y_{1}, \ldots, Y_{N}\right)$, then there is a differential field extension $L$ of $F$, which has 
a differential solution of $f_{1}=0, \ldots, f_{n}=0, H\left(f_{1}, \ldots, f_{n}\right) \neq 0$ such that $L$ is an elementary extension of $F$ as a pure field.

In order to see the claim, we first use 6.1. Let $\mathfrak{p}:=\left[f_{1}, \ldots, f_{n}\right]: H\left(f_{1}, \ldots, f_{n}\right)^{\infty}$. Since $\left(f_{1}, \ldots, f_{n}\right)$ is a characteristic set of a differential prime ideal, this prime ideal is p. By [6.1, the differential $F$-algebra $A:=F\{Y\} / \mathfrak{p}$ localized at $h$ is $F$-isomorphic to $B_{h} \otimes_{F} P$, where $B=A\left(f_{1}, \ldots, f_{n}\right) /\left(\mathfrak{p} \cap A\left(f_{1}, \ldots, f_{n}\right)\right)$ and $P$ is a polynomial ring over $F$ in at most countably many variables. Now $\mathfrak{p} \cap A\left(f_{1}, \ldots, f_{n}\right)$ is the ideal $\left(f_{1}, \ldots, f_{n}\right): H\left(f_{1}, \ldots, f_{n}\right)^{\infty}$ of $A\left(f_{1}, \ldots, f_{n}\right)$. Since $\left(f_{1}, \ldots, f_{n}\right)$ is an algebraically prepared system, the ideal $\left(f_{1}, \ldots, f_{n}\right): H\left(f_{1}, \ldots, f_{n}\right)^{\infty}$ has a regular, $F$-rational point. Since $F$ is large, this means that the pure field $F$ is existentially closed in $B$ by 5.3 Thus the pure field $F$ is existentially closed in $B_{h}$, too. Consequently there is a field $L_{0}$ containing $B_{h}$, which is an elementary extension of the pure field $F$. Let $L \succ L_{0}$ be a field of infinite transcendence degree over $L_{0}$. Then, there is an $F$-embedding of $P$ into $L$ so that the indeterminates of $P$ are mapped onto algebraically independent elements of $L$ w.r.t $L_{0}$. Since $A_{h}=B_{h} \otimes_{F} P, A_{h}$ can be embedded into $L$ over $F$. Finally we can extend the derivatives of $A_{h}$ to commuting derivatives of $L$, and we get a differential field as desired.

From the claim, we get assertion (II) by transfinite induction. This is possible because any algebraically prepared system of $F$ is again an algebraically prepared system of $L$, for every differential field $L \supseteq F$ with the property that $F$ is algebraically closed in $L$, in particular if $F \prec L$ as pure fields. Moreover, if $L$ is such a differential field, then $L$ is again large by 5.2 Hence we can iterate the claim until all algebraically prepared systems of the differential fields constructed so far are solvable in the union of all these differential fields. So this union will be a model of $\mathrm{UC}_{K}$ and an elementary extension of $F$ when viewed as a pure field.

Remark. If $F$ is a large field and $f_{1}, \ldots, f_{n}, h \in F[X], X=\left(X_{1}, \ldots, X_{k}\right)$ such that $\left(f_{1}, \ldots, f_{n}\right): h^{\infty}$ is a prime ideal which has a regular $F$-rational point, then it also has a regular $F$-rational point where $h$ does not vanish. The reason is that the generic point of $\left(f_{1}, \ldots, f_{n}\right): h^{\infty}$ has this property and $F$ is existentially closed in $F /\left(f_{1}, \ldots, f_{n}\right): h^{\infty}$.

Therefore, for our purposes, in condition (AP2) of 3.1 one can drop the term "where $H\left(f_{1}, \ldots, f_{n}\right)$ does not vanish".

(6.3) Proposition. The theory of large fields, the theory $\mathrm{UC}_{K}$ and the theory $\mathrm{UC}_{K} \cup$ "large fields" are inductive. If $U$ is another theory of large differential fields in $K$ commuting derivatives, such that properties (I) and (II) of 6.2 hold for $U$, then $U$ contains $\mathrm{UC}_{K}$. If in addition $U$ is inductive, then $U=\mathrm{UC}_{K} \cup$ "large fields".

Proof. An increasing chain of large fields is again a large field. This follows easily from the definition. Also, from the definition of $\mathrm{UC}_{K}$, an increasing chain of models of $\mathrm{UC}_{K}$ is again a model of $\mathrm{UC}_{K}$. By classical model theory, this is equivalent to the inductivity of the corresponding theories.

Let $U$ be another theory of large differential fields, such that properties (I) and (II) of 6.2 hold for $U$. In order to show $\mathrm{UC}_{K} \subseteq U$ we take a model $M$ of $U$ and we have to show that $M$ is a model of $\mathrm{UC}_{K}$. Since $M$ is a large field, property (II) of 6.2 says that there is a differential field $M \subseteq N \models \mathrm{UC}_{K}$ such that $M \uparrow \mathcal{L} \prec N \uparrow \mathcal{L}$. Again $N$ is a large field and property (II) of 6.2 applied to $U$ gives a differential field $N \subseteq M^{\prime} \models U$ with $N \uparrow \mathcal{L} \prec M^{\prime} \uparrow \mathcal{L}$. It follows that $M \uparrow \mathcal{L} \prec M^{\prime} \uparrow \mathcal{L}$ and 
since property (I) of 6.2 is satisfied for $U$, we get that $M$ is existentially closed in $M^{\prime}$. Consequently $M$ is existentially closed in $N$, too. Since $N \models \mathrm{UC}_{K}$ and $\mathrm{UC}_{K}$ is inductive, it follows that $M \models \mathrm{UC}_{K}$.

This shows that $\mathrm{UC}_{K} \subseteq U$. If in addition $U$ is inductive, then the same argument as above with interchanged roles of $U$ and $\mathrm{UC}_{K} \cup$ "large fields" implies equality of both theories.

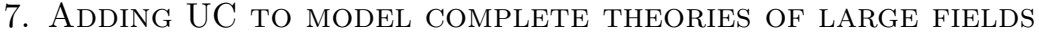

Recall from $\mathrm{Ho}$ that in an arbitrary language $\mathcal{L}$, an $\mathcal{L}$-theory $T$ is a model companion of another $\mathcal{L}$-theory $T_{0}$, if $T$ and $T_{0}$ have the same universal theory and if $T$ is model complete. If this is the case and $T_{0}$ has the amalgamation property in addition, then $T$ is called a model completion of $T_{0}$. Moreover both notions can be characterized as follows.

For an $\mathcal{L}$-structure $M$ let $\mathcal{L}(M)$ denote the language obtained from $\mathcal{L}$ by adding a new constant $\underline{m}$ for each element in $M$. Moreover let $\operatorname{diag}(M)$ be the diagram of $M$, thus the set of all quantifier-free $\mathcal{L}(M)$-sentences that are true in $M$, when we interpret the $\underline{m}$ as $m$.

Let $T_{0}, T$ be $\mathcal{L}$-theories with the same universal theory. Hence every model of $T_{0}$ can be embedded into a model of $T$ and vice versa. Then

$T$ is a model companion of $T_{0}$ if and only if for every model $M$ of $T$, the theory $T \cup \operatorname{diag}(M)$ is a complete $\mathcal{L}(M)$-theory.

$T$ is a model completion of $T_{0}$ if and only if for every model $M$ of $T_{0}$ and every model $M$ of $T$, the theory $T \cup \operatorname{diag}(M)$ is a complete $\mathcal{L}(M)$-theory.

$T$ has quantifier elimination if and only if for every substructure $A$ of a model $M$ of $T$ the theory $T \cup \operatorname{diag}(A)$ is a complete $\mathcal{L}(A)$-theory.

Hence $T$ has quantifier elimination $\Rightarrow T$ is a model completion of $T_{0} \Rightarrow T$ is a model companion of $T_{0}$.

Recall also that a given $\mathcal{L}$-theory $T_{0}$ has at most one model companion if $T_{0}$ is axiomatized by $\forall_{2}$-sentences.

If $T$ is a model complete theory, then it is well known that $T$ is axiomatized by $\forall_{2}$-sentences. Hence $T$ is the unique model companion of $T$ and $T$ is the model completion of $T$.

The theory $\mathrm{UC}_{K}$ serves as a uniform model companion, a uniform model completion and a uniform model completion with quantifier elimination of theories of differential fields which are companionable in the various senses as theories of pure fields, in the following sense:

(7.1) Theorem. Let $K \in \mathbb{N}$. Let $\mathcal{L}:=\{+,-, \cdot, 0,1\}$ be the language of rings and let $C$ be a set of new constants. Let $\partial_{1}, \ldots, \partial_{K}$ be new unary function symbols. Let $T$ be a model complete theory in the language $\mathcal{L}(C)$ such that every model of $T$ is a large field.

Let $T^{*}$ be a theory in a language $\mathcal{L}^{*} \supseteq \mathcal{L}(C)$ such that $T^{*}$ contains $T$ and $T^{*}$ is an extension by definitions of $T$. This means that $T^{*}$ is built from $T$ by saying that each new symbol of $\mathcal{L}^{*}$ is equivalent to a certain $\mathcal{L}(C)$-formula. Nothing else is added in $T^{*}$.

Let $A$ be an $\mathcal{L}^{*}(\bar{\partial})$-structure, $\bar{\partial}:=\left(\partial_{1}, \ldots, \partial_{K}\right)$ such that $A$, viewed as an $\mathcal{L}(\bar{\partial})$ structure, is a differential ring in $K$ commuting derivatives.

If $T^{*} \cup \operatorname{diag}\left(A\left\lceil\mathcal{L}^{*}\right)\right.$ is complete, then $T^{*} \cup \mathrm{UC}_{K} \cup \operatorname{diag}(A)$ is complete. 
Proof.

Claim 1. $T^{*} \cup \mathrm{UC}_{K} \cup \operatorname{diag}(A)$ is consistent.

Since $T^{*} \cup \operatorname{diag}\left(A\left\lceil\mathcal{L}^{*}\right)\right.$ is consistent, $A\left\lceil\mathcal{L}^{*}\right.$ is a substructure of a model of $T^{*}$. Since the derivatives of $A$ can be extended to commuting derivatives of that model, we get that $T^{*} \cup \operatorname{diag}(A) \cup$ "differential fields" is consistent. By 6.2(II) and since $T^{*}$ is an extension by definition of $T, T^{*} \cup \mathrm{UC}_{K} \cup \operatorname{diag}(A)$ is also consistent.

Before proving that $T^{*} \cup \mathrm{UC}_{K} \cup \operatorname{diag}(A)$ is complete we first prove that $T^{*} \cup \mathrm{UC}_{K}$ is model complete and that $T^{*} \cup \mathrm{UC}_{K}$ has quantifier elimination if $T^{*}$ has quantifier elimination. So let us forget $A$ for the moment.

Claim 2. $T^{*} \cup \mathrm{UC}_{K}$ is model complete.

First note that $T \cup \mathrm{UC}_{K}$ is consistent, since for every model $M$ of $T, M$ can be equipped with $K$ commuting derivatives. Thus by Claim $1, T \cup \mathrm{UC}_{K}$ is consistent. $T \cup \mathrm{UC}_{K}$ is model complete by 6.2(I) and the Robinson test for model completeness. Since $T^{*}$ is an extension by definitions of $T, T^{*} \cup \mathrm{UC}_{K}$ is also model complete (and consistent). This shows Claim 2.

Claim 3. If $T^{*}$ has quantifier elimination, then $T^{*} \cup \mathrm{UC}_{K}$ has quantifier elimination.

In order to see this let $M, N \models T^{*} \cup \mathrm{UC}_{K}$ and let $A \subseteq M, N$ be a common $\mathcal{L}^{*}$ substructure, which is also a common differential subring of $M, N$. Let $\varphi(\bar{x}, \bar{u})$ be a quantifier-free $\mathcal{L}^{*}(\bar{\partial})$-formula, $\bar{a} \in A^{\bar{u}}$, such that $M=\varphi(\bar{b}, \bar{a})$ for some $\bar{b} \in M^{\bar{x}}$. As $T^{*}$ has quantifier elimination we know that $M \equiv_{A} N$ as $\mathcal{L}^{*}$-structures. In particular $M \equiv_{\exists, A} N$ as pure fields. Since $M, N \models \mathrm{UC}_{K}, 6$. 6 (I) implies $M \equiv_{\exists, A} N$ in the language $\mathcal{L}(C)(\bar{\partial})$ (observe that $M$ and $N$ interpret the constants from $C$ as the same elements from $A)$.

Since $T \cup \mathrm{UC}_{K}$ is a model complete $\mathcal{L}(C)(\bar{\partial})$-theory and $T^{*} \cup \mathrm{UC}_{K}$ is an extension by definition of $T \cup \mathrm{UC}_{K}$, there is a quantifier-free formula $\chi(\bar{x}, \bar{u}, \bar{y})$ in the language $\mathcal{L}(C)(\bar{\partial})$, such that $T^{*} \cup \mathrm{UC}_{K} \vdash \forall \bar{x}, \bar{u}(\varphi(\bar{x}, \bar{u}) \leftrightarrow \exists \bar{y} \chi(\bar{x}, \bar{u}, \bar{y}))$. Since $M=\varphi(\bar{b}, \bar{a})$ and $M=T^{*} \cup \mathrm{UC}_{K}$, there is some $\bar{b}^{\prime} \in M^{\bar{y}}$ such that $M \models \chi\left(\bar{b}, \bar{a}, \bar{b}^{\prime}\right)$. As $M \equiv_{\exists, A} N$ as differential fields, there are $\bar{c}, \bar{c}^{\prime} \subseteq N$ such that $N \models \chi\left(\bar{c}, \bar{a}, \bar{c}^{\prime}\right)$, hence $N \models \exists \bar{y} \chi(\bar{c}, \bar{a}, \bar{y})$. As $N \models T^{*} \cup \mathrm{UC}_{K}, N \models \varphi(\bar{c}, \bar{a})$ as desired. This shows Claim 3.

Now we prove the theorem. Since $T^{*} \cup \operatorname{diag}\left(A\left\lceil\mathcal{L}^{*}\right)\right.$ is consistent, $T^{*} \cup \mathrm{UC}_{K} \cup$ $\operatorname{diag}(A)$ is consistent by Claim 1 .

Let $M, N$ be two models of $T^{*} \cup \mathrm{UC}_{K}$ and let $A$ be a common $\mathcal{L}^{*}(\bar{\partial})$-substructure. We have to show that $M \equiv_{A} N$. Let $\mathcal{L}^{+}$be the language containing $\mathcal{L}^{*}$ together with a new $k$-ary relation $R_{\varphi}$ for every existential $\mathcal{L}^{*}$-formula $\varphi$ in exactly $k$ free variables. Let $T^{+}$be the $\mathcal{L}^{+}$-theory containing $T^{*}$ and all the sentences $\forall u_{1}, \ldots, u_{k} R_{\varphi}(\bar{u}) \leftrightarrow \varphi(\bar{u})$, where $\varphi=\varphi(\bar{u})$ runs through all existential $\mathcal{L}^{*}$-formulas in exactly $k$ free variables $\bar{u}=\left(u_{1}, \ldots, u_{k}\right), k \in \mathbb{N}_{0}$.

Since $T^{*}$ is an extension by definition of $T, T^{+}$is also an extension by definition of $T$ and of $T^{*}$. Since $T$ is model complete, $T^{+}$is model complete, too. It follows that $T^{+}$has quantifier elimination. By Claim $3, T^{+} \cup \mathrm{UC}_{K}$ has quantifier elimination.

Let $\tilde{M}, \tilde{N}$ be the expansions of $M, N$ to models of $T^{+} \cup \mathrm{UC}_{K}$, respectively, according to the axioms of $T^{+}$. Since $A$ is a common $\mathcal{L}^{*}(\bar{\partial})$-substructure of $M$ and $N$, the differential ring $A$ is a common differential subring of $M$ and $N$. Since 
$T^{*} \cup \operatorname{diag}\left(A\left\lceil\mathcal{L}^{*}\right)\right.$ is complete we know that $M\left\lceil\mathcal{L}^{*} \equiv_{A} N\left\lceil\mathcal{L}^{*}\right.\right.$. It follows from the definition of $T^{+}$that $A$ together with the substructure induced by $\tilde{M}$ on $A$ is the same as $A$ together with the substructure induced by $\tilde{N}$ on $A$. Hence $\tilde{M}$ and $\tilde{N}$ induce the same structure on $A$, and the quantifier elimination of $T^{+} \cup \mathrm{UC}_{K}$ implies $\tilde{M} \equiv_{A} \tilde{N}$. But then $M \equiv_{A} N$, too.

The next theorem gathers consequences of [7.1.

(7.2) Theorem. Let $K \in \mathbb{N}$. Let $\mathcal{L}:=\{+,-, \cdot, 0,1\}$ be the language of rings and let $C$ be a set of new constants. Let $\partial_{1}, \ldots, \partial_{K}$ be new unary function symbols. Let $T$ be a model complete theory in the language $\mathcal{L}(C)$ such that every model of $T$ is a large field.

Let $T^{*}$ be a theory in a language $\mathcal{L}^{*} \supseteq \mathcal{L}(C)$ such that $T^{*}$ contains $T$ and $T^{*}$ is an extension by definitions of $T$.

Assume that $T^{*}$ is a model companion of an $\mathcal{L}^{*}$-theory $T_{0}^{*}$ extending the theory of fields. Then:

(i) $T^{*} \cup \mathrm{UC}_{K}$ is a model companion of the $\mathcal{L}^{*}(\bar{\partial})$-theory $T_{0}^{*} \cup$ "differential fields in K commuting derivatives".

(ii) If $T^{*}$ is a model completion of $T_{0}^{*}$, then $T^{*} \cup \mathrm{UC}_{K}$ is a model completion of the $\mathcal{L}^{*}(\bar{\partial})$-theory $T_{0}^{*} \cup$ "differential fields in $K$ commuting derivatives".

(iii) If $T^{*}$ has quantifier elimination, then $T^{*} \cup \mathrm{UC}_{K}$ has quantifier elimination.

(iv) If $T$ is complete and $M$ is a differential field and a model of $T$, then $T^{*} U$ $\mathrm{UC}_{K} \cup \operatorname{diag}(\mathcal{C})$ is complete, where $\mathcal{C}$ is the $\mathcal{L}(C)(\bar{\partial})$-substructure generated by $\emptyset$ in $M$ (so $\mathcal{C}$ is the differential subring of $M$ generated by $c^{M}, c \in C$ ).

Proof. We first show that $T_{0}^{*} \cup$ "differential fields" has the same universal theory as $T^{*} \cup \mathrm{UC}_{K}$.

First let $M$ be a model of $T^{*} \cup \mathrm{UC}_{K}$. By assumption, there is an $\mathcal{L}^{*}$-embedding $M \uparrow \mathcal{L}^{*} \longrightarrow N_{0}$ of $M \uparrow \mathcal{L}^{*}$ into a model $N_{0}$ of $T_{0}^{*}$. If we extend the derivatives of $M$ to $N_{0}$ we get a model $N$ of $T_{0}^{*} \cup$ "differential fields", and the given embedding is an $\mathcal{L}^{*}(\bar{\partial})$-embedding, too.

Conversely let $M \models T_{0}^{*} \cup$ "differential fields". By assumption there is an $\mathcal{L}^{*}$ embedding $M \uparrow \mathcal{L}^{*} \longrightarrow N_{0}$ of $M \uparrow \mathcal{L}^{*}$ into a model $N_{0}$ of $T^{*}$. Extend the derivatives of $M$ to $N_{0}$ and call the resulting $\mathcal{L}^{*}(\bar{\partial})$-structure $N_{1}$. By 6.2(II), there is a model $L$ of $T \cup \mathrm{UC}_{K}$, such that $L$ is a differential field extension of $N_{1} \uparrow \mathcal{L}(C)(\bar{\partial})$. Now extend the new symbols of $\mathcal{L}^{*}$ according to the rules in $T^{*}$ to $L$ and call the resulting $\mathcal{L}^{*}(\bar{\partial})$-structure $N$. Then $M \longrightarrow N$ is an embedding of $\mathcal{L}^{*}\left(\partial_{1}, \ldots, \partial_{K}\right)$-structures and $N \models T^{*} \cup \mathrm{UC}_{K}$. This shows that $T_{0}^{*} \cup$ "differential fields" has the same universal theory as $T^{*} \cup \mathrm{UC}_{K}$.

Now items (i)-(iii) of the theorem follows from 7.1 and the characterization of the various companions at the beginning of this section. Item (iv) also follows from 7.1. since $T^{*}$ is complete if $T$ is complete.

I do not know if every model complete field (of characteristic 0 ) in the language of rings is large. Here, a model complete field is a field $F$ such that the theory of $F$ in the language of rings is model complete.

The following remark on the axiomatizability of theories with quantifier elimination shows that for each particular $T^{*}$ as in 7.2(iii) and each given number $K$ of derivatives, one can expect an easier axiom system for $T^{*} \cup \mathrm{UC}_{K}$ than the one given in 4.5 , 
(7.3) Remark. Let $T$ be a theory in an arbitrary language $\mathcal{L}$ and suppose $T$ has quantifier elimination. Then $T$ has an axiom system consisting of $\mathcal{L}$-sentences of the form

$$
\forall \bar{x} \exists y \varphi(\bar{x}, y),
$$

where $y$ is a single variable and $\varphi(\bar{x}, y)$ is a quantifier-free $\mathcal{L}$-formula.

Proof. Let $\Sigma:=\{\forall \bar{x} \exists y \varphi(\bar{x}, y) \in T \mid \varphi(\bar{x}, y)$ quantifier free $\}$. Let $M=\Sigma$. We have to show that $M \models T$. Since $M \models T_{\forall}$, there is a model $N$ of $T$, such that $M$ is a substructure of $N$, and it is enough to prove that $M \prec N$. To see this we use the Tarski-Vaught test. This means we have to take an arbitrary $\mathcal{L}$-formula $\psi(\bar{x}, y)$, where $y$ is a single variable together with some $\bar{a} \in M^{\bar{x}}$ such that $N \models \exists y \psi(\bar{a}, y)$, and we have to find some $b \in M$ such that $N \models \psi(\bar{a}, b)$. Since $T$ has quantifier elimination we may assume that $\psi(\bar{x}, y)$ is quantifier free. Moreover there is a quantifier-free $\mathcal{L}$-formula $\chi(\bar{x})$ such that $T \vdash \forall \bar{x}[\chi(\bar{x}) \leftrightarrow \exists y \psi(\bar{x}, y)]$. Hence

$$
T \vdash \forall \bar{x} \exists y[\chi(\bar{x}) \rightarrow \psi(\bar{x}, y)] .
$$

This sentence is in $\Sigma$, thus $M \models \forall \bar{x} \exists y[\chi(\bar{x}) \rightarrow \psi(\bar{x}, y)]$. As $N \models \chi(\bar{a})$ and $\chi$ is quantifier free we have $M \models \chi(\bar{a})$. Hence there is some $b \in M$ such that $M \models \psi(\bar{a}, b)$, consequently $N \models \psi(\bar{a}, b)$.

\section{EXAMPLES}

(8.1) Proposition. Suppose $F$ is a field and $F$ has one of the following properties:

(1) $F$ is pseudo algebraically closed $(P A C)$ or $F$ is algebraically closed $(A C F)$ or

(2) $F$ is pseudo real closed (PRC) or $F$ is real closed (RCF) or

(3) $F$ is pseudo p-adically closed of fixed rankd $\left(P p C_{d}\right)$ or $F$ is $p$-adically closed of fixed rank $d\left(p C_{d}\right)$ or

(4) F admits a nontrivial henselian valuation.

Then $F$ is a large field.

Proof. (1)-(3) can be found in $[\mathrm{Po}]$. Proposition 3.1 of $[\mathrm{Po}]$ provides a more general source of large fields, which contain those in (1)-(3): Every field which satisfies a universal local-global principle (cf. [Po], section 3A) is large.

(4) follows from [PZ, thm. 7.4: Since a henselian-valued field satisfies an implicit function theorem, every smooth integral curve defined over $F$ which has an $F$ rational point, has infinitely many $F$-rational points.

Now let $\mathcal{L}$ be the language $\{+,-, \cdot, 0,1\}$ of rings again. Recall that the theory of algebraically closed fields of characteristic $0\left(\mathrm{ACF}_{0}\right)$, the theory of real closed fields $(\mathrm{RCF})$ and the theory of p-adically closed fields of fixed rank $d\left(\mathrm{pC}_{d}\right)$ are model complete. In Wh1] and Wh2] a broad class of model complete $\mathcal{L}$-theories of $P A C$, $P R C$ and $P p C$ fields can be found.

\section{(8.2) Corollary and Definition.}

(i) $A C F_{0} \cup \mathrm{UC}_{K}$ is the theory of differentially closed fields as introduced by [Bö] and [McG] in the partial case $K \geq 1$ and by L. Blum in the ordinary case $K=1$. 
(ii) $R C F \cup \mathrm{UC}_{K}$ is the complete and model complete theory of real closed ordered differential fields in the language $\mathcal{L}\left(\partial_{1}, \ldots, \partial_{K}\right)$. This theory was introduced in the ordinary case $K=1$ by $M$. Singer in [Si]. Moreover $R C F \cup \mathrm{UC}_{K}$ has quantifier elimination in the language $\mathcal{L}(\leq)\left(\partial_{1}, \ldots, \partial_{K}\right)$.

(iii) $p C_{d} \cup \mathrm{UC}_{K}$ is the complete and model complete theory of p-adically closed differential fields of fixed rank $d$ in the language $\mathcal{L}\left(\partial_{1}, \ldots, \partial_{K}\right) ; p C_{d} \cup \mathrm{UC}_{K}$ has quantifier elimination in the language $\mathcal{L}\left(P_{n} \mid n \in \mathbb{N}\right)\left(\partial_{1}, \ldots, \partial_{K}\right)$, where $P_{n}$ is a unary predicate for the $n$-th powers of the field.

Proof. By 7.2 and the corresponding results for the underlying theories of fields by Chevalley, Tarski and Macintyre.

Note also, that in $[\mathrm{PP}$ (for $K=1$ ) and in [Pi] (for $K \geq 1$ ) reformulations of $A C F_{0} \cup \mathrm{UC}_{K}$ in terms of properties of ordinary varieties can be found.

Another application of Theorems 7.1 and 7.2 within a special class of PAC-fields concerns pseudo finite fields of characteristic 0 . Recall that a pseudo finite field is a PAC-field which has exactly one extension of degree $n$ for every $n \in \mathbb{N}$. The theory of a given pseudo finite field $F$ is not model complete in general. But it is model complete after naming some elements of the field by new constants:

(8.3) Proposition. For each $n \in \mathbb{N}, n>1$, let $c_{n 1}, \ldots, c_{n n}$ be new constants and let $C:=\left\{c_{n i} \mid i \leq n \in \mathbb{N}, n>1\right\}$. Let $T$ be the theory of pseudo finite fields together with the $\mathcal{L}(C)$-sentences which express that the polynomial $T^{n}+c_{n 1} T^{n-1}+\ldots+c_{n n}$ is irreducible, $n>1$. Then $T$ is a model complete $\mathcal{L}(C)$-theory.

Proof. [CDM], Proposition (2.7), which contains a more detailed elimination result.

The theories of all models of the $\mathcal{L}(C)$-theory $T$ from 8.3 of characteristic 0 is denoted by $\operatorname{Psf}_{C}(0)$.

(8.4) Corollary. The $\mathcal{L}(C)\left(\partial_{1}, \ldots, \partial_{K}\right)$-theory $T=P s f_{C}(0) \cup \mathrm{UC}_{K}$ is the model completion of the theory $P s f_{C}(0) \cup$ "differential fields in $K$ commuting derivatives". Moreover, if $(F, C) \models T$ and $F$ is algebraic over $\mathbb{Q}$, then $T h(F, C) \cup \mathrm{UC}_{K}$ is complete.

Proof. $\mathcal{L}(C)\left(\partial_{1}, \ldots, \partial_{K}\right)$-theory $\operatorname{Psf}_{C}(0) \cup \mathrm{UC}_{K}$ is the model completion of the theory $\operatorname{Psf}_{C}(0) \cup$ "differential fields in $K$ commuting derivatives", by 8.1 (1), 8.3 and 7.2(ii). For the moreover part we use 7.2 (iv); observe that in every model of $T h(F, C) \cup \mathrm{UC}_{K}$ the derivatives on $C$ are trivial.

\section{REFERENCES}

[Bö] J. Böger, Modelltheorie von Körpern mit paarweise kommutierenden Derivationen. Diplomarbeit, Freiburg, September 1996.

[CDM] Z. Chatzidakis, L. van den Dries, A. Macintyre, Definable sets over finite fields. J. reine angew. Math. 427 (1992), 107-135. MR1162433 (94c:03049)

[DL] J. Denef, L. Lipshitz, Power Series Solutions of Algebraic Differential Equations. Math. Ann. 267 (1984), 213-238. MR0738249 (85j:12010)

[vdD-Sch] L. van den Dries, K. Schmidt, Bounds in the theory of polynomial rings over fields. A nonstandard approach. Invent. Math. 76 (1984), no. 1, 77-91. MR0739626|(85i:12016)

[E] D. Eisenbud, Commutative Algebra with a View Toward Algebraic Geometry. Springer Graduate Texts in Mathematics 150, 1995. MR1322960 (97a:13001) 
[GaMi] G. Gallo, B. Mishra, Efficient Algorithms and Bounds for the Wu-Ritt Characteristic Sets. Effective methods in algebraic geometry (Castiglioncello, 1990), 119-142, Progr. Math., 94, Birkhäuser-Boston, Boston, MA, 1991. MF1106418 (92e:14058)

[Ho] W. Hodges, Model Theory. Encyclopedia of Mathematics and its Applications, vol. 42, 1993. MR 1221741 (94e:03002)

[Hu] E. Hubert, Factorization-free Decomposition Algorithms in Differential Algebra. J. Symbolic Computation 29 (2000), 641-662. MR1769659 (2001k:12013)

[Ko] E. R. Kolchin, Differential Algebra and Algebraic Groups. Pure and Applied Mathematics, vol. 54, Academic Press, 1973. MR0568864 (58:27929)

[McG] T. McGrail, The model theory of differential fields with finitely many commuting derivations. J. Symbolic Logic 65 (2000), no. 2, 885-913. MR1771092 (2001h:03066)

[Pi] D. Pierce, Differential Forms in the Model Theory of Differential Fields. J. Symbolic Logic 68 (2003), no. 3, 923-945. MR 2000487 (2004h:03080)

[PP] D. Pierce, A. Pillay, A note on the axioms for differentially closed fields of characteristic zero. J. Algebra 204 (1998), no. 1, 108-115. MR1623945 (99g:12006)

[Po] F. Pop, Embedding problems over large fields. Ann. of Math. (2) 144 (1996), no. 1, 1-34. MR:1405941 (97h:12013)

[PZ] A. Prestel, M. Ziegler, Model-theoretic methods in the theory of topological fields. J. Reine Angew. Math. 299(300) (1978), 318-341. MF0491852 (80f:54034)

[Si] M. Singer, The Model Theory of Ordered Differential Fields. The Journal of Symbolic Logic, Vol. 43, Number 1, March 1978, pp. 82-91. MR0495120 (80a:03044)

[Tr] M. Tressl, A Structure Theorem for Differential Algebras. Banach Center Publications, vol. 58, Warszawa 2002, pp. 201-206. MR 1972455 (2004b:12011)

[Wh1] W. H. Wheeler, Model complete theories of formally real fields and formally $p$-adic fields. J. Symbolic Logic 48 (1983), no. 4, 1130-1139 (1984). MR0727801 (85b:03055)

[Wh2] W. H. Wheeler, Model complete theories of pseudo-algebraically closed fields. Ann. Math. Logic 17 (1979), no. 3, 205-226. MR0556892 (81c:03024)

NWF-I Mathematik, 93040 Universität Regensburg, Germany

E-mail address: marcus.tressl@mathematik.uni-regensburg.de 